\title{
Polytraumatized patient with two columns fracture of the acetabulum and skin abrasion, which incision to use with soft tissue: A case report at 15 years follow-up
}

\author{
R. Spagnolo ${ }^{*}$, D. Porreca ${ }^{2}$, M. Vimercati ${ }^{3}$, F. Pace ${ }^{4}$ \\ 'Department of Orthopaedic Surgery, Hospital Niguarda Cà Granda, Milan, Italy \\ ${ }^{2}$ Physiotherapist, ASST BergamomOvest-Hospital, Treviglio Caravaggio, Italy \\ ${ }^{3}$ Physiotherapist, Hospital Niguarda Cà Granda, Milan, Italy \\ ${ }^{4}$ First Orthopaedic Clinic-Hospital Gaetano Pini, University of Milan, Italy
}

\section{Article Info}

\section{Article Notes}

Received: April 09, 2021

Accepted: July 13, 2021

\section{*Correspondence:}

${ }^{*}$ Dr. R. Spagnolo, Department of Orthopaedic Surgery, Hospital Niguarda Cà Granda, Milan, Italy;

Email: rosariospagnolo@libero.it

${ }^{0} 2021$ Spagnolo R. This article is distributed under the terms of the Creative Commons Attribution 4.0 International License.

\section{Keywords}

Acetabulum

Burns

Posterior approach

Two column bus

Minimally invasive

\section{Abstract}

Posterior fractures of the acetabulum are the most frequent pattern of acetabular fractures. Based on the works of Judet and Letournel, accurate reduction and stable osteosynthesis with early mobilization have become the gold standard for the treatment of posterior acetabular fractures. Recently, a less invasive approach has been described to decrease Kocher-Langenbeck (K-L) approach complications. The clinical case describes a patient affected by two-column fractures of the acetabulum with skin abrasions of the gluteus. The clinical evaluation was based on Merle d'Aubigne and Postel scoring, which Matta has modified. The approach used is a modified, less invasive K-L: a straight skin incision from the midpoint between the posterior superior iliac spine and the posterior tip of the greater trochanter. We observed the essential advantages using this approach were a lesser split of the gluteus maximus and no risk of damage for the superior gluteal nerve. In the early postoperative rehabilitation, we examined the strength of the gluteus maximus, which was better than in patients treated with the typical Kocher-Langenbeck approach. The patient resumed after fifteen years after surgery; hip mobility is complete and painless.

\section{Introduction}

Over recent decades, minimally-invasive surgery has become a clinical trend because of its expected benefits, including less tissue trauma and early rehabilitation. Accurate reduction and stable osteosynthesis with early mobilization have become the gold standard for treating posterior acetabular fractures, as described by Judet and Letournel ${ }^{1,2}$. In recent years, orthopedic surgery has focused on minimizing the surgical approach and reducing the damage caused by invasive surgery. One of the risks of surgical approaches to both columns of the acetabulum is to damage the superior gluteal artery and nerve. Many studies have shown that heterotopic ossification ( $\mathrm{HO}$ ) incidence is more significant in acetabular fracture surgery. This fact has been attributed to extended surgical approaches ${ }^{3,4}$. In the last decade, new surgical approaches have been proposed to reduce complications with the traditional K-L. In recent reports, new procedures have been described. The SER-sparing modifications of the K-L approach for selected posterior acetabular fractures have been reported by Sarlak $^{3}$ and Magu ${ }^{4}$. J. B. Carr and P. B. Leach ${ }^{5}$ and Spagnolo R. et al. ${ }^{6}$ have described a K-L modification to reduce posterior acetabular fractures. 
Moed BR in 2010 and 2014,8 reported a lateral approach in case of skin abrasions.

In this paper, we describe a clinical case of a polytraumatized patient with a two-column fracture of the right acetabulum, skin abrasion of his lower back and proximal right thigh, and an open fracture of his right hand and left foot. This paper aims to describe the surgical approach used to reduce the posterior acetabular fracture paying attention to skin lesions.

\section{Materials and Methods}

The patient was studied with pre-operative X-rays and CT scan with 3D reconstruction to better plan the surgery (Figure 1). X-ray series includes AP view, iliac oblique view, and obturator oblique view (Judet views). The surgical treatment of the acetabular fracture was carried out in two stages, when general conditions were stabilized. The clinical evaluation was based on Merle d'Aubigne and Postel scoring, which has been modified by Matta ${ }^{9}$. According to this clinical grading system, pain, gait, and range of motion of the hip assign a maximum of six points each; the three individual scores were summed to derive the final scores. The clinical result was classified as excellent (18 points), very good (17 points), good (15 or 16 points), fair (13 or 14 points), or poor ( $<13$ points). The radiographs were evaluated according to the criteria developed by Matta. According to radiographic criteria, a grade of excellent indicates a normal appearance of the hip, a grade of good changes, small osteophytes; moderate $(1 \mathrm{~mm})$ narrowing of the joint, and minimum sclerosis, grade of fair means intermediate changes, moderate osteophytes, moderate (less 50\%) narrow joint, and moderate sclerosis; grade of poor, advanced changes, large osteophytes, severe narrowing of the joint, collapse or wear of the femoral head and acetabular wear. We assessed the post-operative reduction by taking three standard plain radiographs of the pelvis (anteroposterior and two $45^{\circ}$ oblique Judet views). We evaluated fracture reduction by measuring the residual displacement in millimeters; it was classified as anatomical $(0-1 \mathrm{~mm}$ of displacement), imperfect $(2-3 \mathrm{~mm})$, and poor ( $>3 \mathrm{~mm})$. Heterotopic ossification was classified according to Brooker. The patient received a daily dose of LMWH as anti-thrombotic prophylaxis e antibiotics.

\section{Clinical History}

In May 2006, a 27-year-old male had a motorbike accident against a car, reporting sub amputation of his right hand, right pelvic, and acetabular fracture with cutaneous lesions of his back and proximal right thigh and multiple metatarsals and fingers fractures of the left foot. He also suffered a complete right sciatic nerve injury, thoracic and abdominal trauma, and head concussion without losing consciousness.
He has been operated on an emergency regimen to perform an arthrodesis of the trapezium-metacarpal joint with two crossed K-wires and suture of the thumb's ulnar collateral artery, and multiple tenorrhaphies. The second and fourth fingers have been fixed with two crossed K-wires; the third finger could not be replanted due to severe lesions. A fasciotomy of the flexor compartment of the right forearm was necessary. Covering of the cutaneous defect of the right hand and forearm was performed too. The metatarsal fractures were treated conservatively with a plaster cast and suture of the wounds. Skeletal traction was applied to the right inferior limb. The patient was transferred to our hospital to treat pelvic and acetabular fractures after seven days, once improved the hand lesion and skin abrasion. Pelvic CT scan and x-ray were executed (Figure 1).

\section{Surgery}

We performed the first surgery to stabilize the posterior column and wall of the right acetabulum with the patient prone with the limb in skeletal traction and the knee $90^{\circ}$ flexed to reduced sciatic nerve damage; the nerve was already paretic due to the trauma (Figure 2). The approach we used was a less invasive posterior approach we practice since 2004 to avoid skin abrasions.
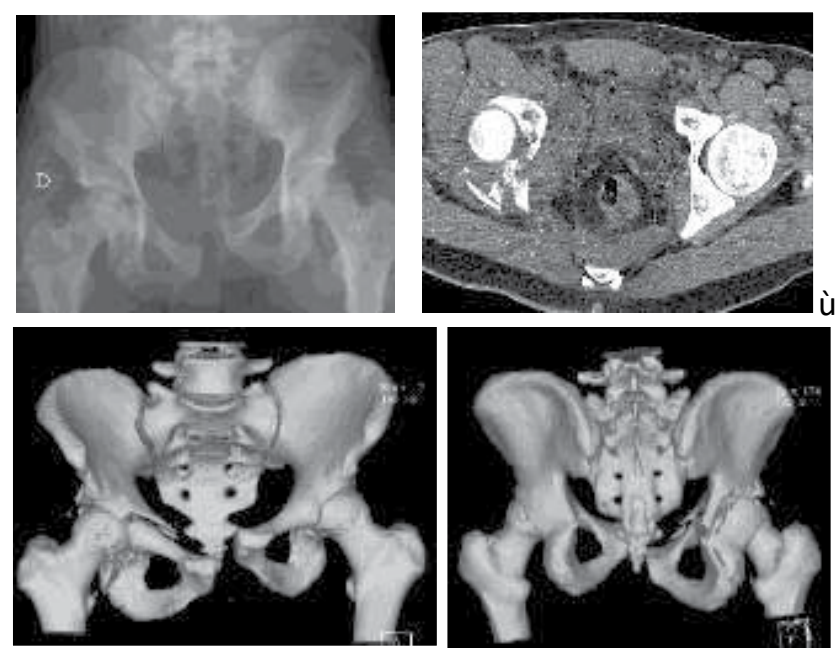

Figure 1: 27-year-old man 2006 fracture. Bicolumn fracture with instability of the right ileo-sacral joint and symphyseal rupture
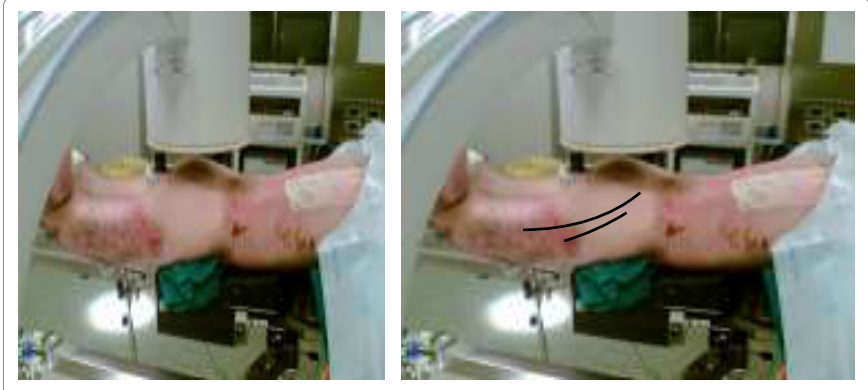

Figure 2: Surgical position, skin abrasion, K-L and minimal access 


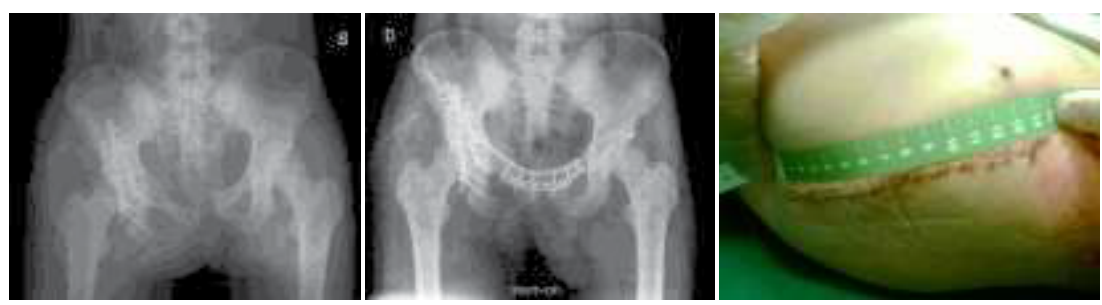

Figure 3: Stepwise post-operative radiographs and skin incision
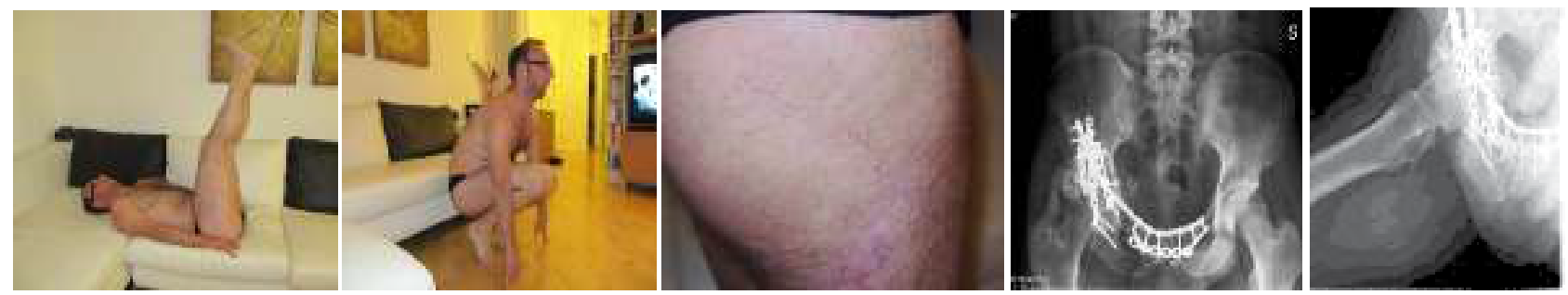

Figure 4: Mobility and radiographs after 8 years

The incision started from the distal part of the K-L approach. It finished $1 \mathrm{~cm}$ proximal to the greater trochanter (Figure 3); the external rotators protected the sciatic nerve, which was in continuity without hematoma. We reduced the posterior wall fracture by positioning a transverse screw and three stainless-steel plates using fewer screws possible to avoid difficulties with the second stage surgery via the ileo-inguinal approach (Figure 4). The first stage surgery took 70 minutes with $350 \mathrm{ml}$ of blood loss.

While waiting for the second stage, we continued to apply the skeletal traction. The wound was healing without complications, and skin abrasions were dressed as indicated by the plastic surgeon.

We have done the second stage surgery seven days after, performing an anterior approach (ileo-inguinal). The pre-operative planning foresees a plate anchored to the contralateral pubic branch to fix the anterior pelvic instability. The plate was fixed with screws in both pubic branches and proximal in the iliac wing (Figure 5), as we usually do in anteroposterior instability. This stage took 2 hours with $500 \mathrm{ml}$ of blood loss. On the second postop day, the patient received two blood transfusions. The patients received a daily dose of LMWH as anti-thrombotic prophylaxis for seven weeks and cephalosporine antibiotic prophylaxis for seven days.

The patient started physiotherapy on the third-day post-op with early mobilization of the hip; it consisted of passive hip motion, strengthening the abductor and gluteal muscles without weight-bearing. Six weeks after surgery (end of July), we discharged the patient to a rehabilitation clinic. The rehab has been long and intensive, and it took four months because of the
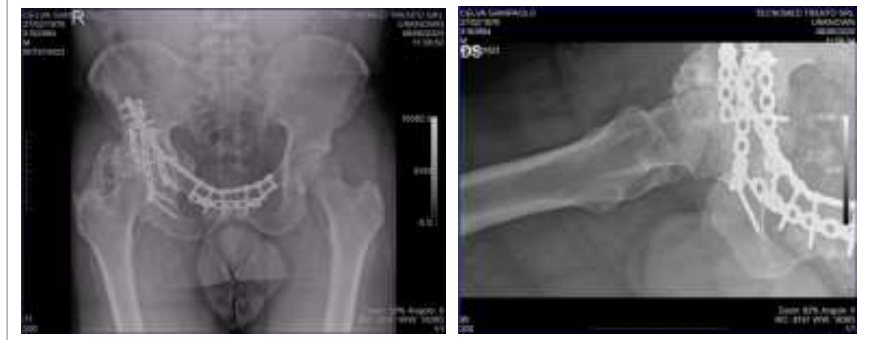

Figure 5: Radiographs after 15 years showing intact hardware, ectopic ossification Brooker 3

difficulties of moving with a wheelchair first and using crutches due to his complex hand injury. Once skin abrasions healed, the rehabilitation continues with pool exercises, starting walking in progressive weight-bearing (for 40 days) when the condition of the hand allowed its use.

In November, the patient was discharged from rehab, walking in full weight-bearing with Codivilla spring, even sciatic nerve injury was improving.

\section{Results}

The result for clinical outcome, according to Merle d'Aubigne and Postel, was classified as excellent. According to the criteria developed by Matta, the post-operative reduction and the latest follow-up were classified as anatomical reduction (Figure 4).

Heterotopic ossification (Brooker's grade 3) did not limit the range of motion of the hip (Figure 5). The paralysis of the sciatic nerve recovered spontaneously in 16 months. No infections or pulmonary embolism complications occurred. After fifteen years, the patient did not present clinical or radiographical signs of arthritis. 


\section{Discussion}

The Orthopedic surgeons use Kocher-Langenbeck's approach to treat fractures of the posterior pelvis. The correlation between extended approaches and $\mathrm{HO}$ is well known in the literature. Perves ${ }^{10}$ described a posterior perigluteal approach with improved muscle tone and decreased ossification rate. The event of superior and inferior gluteal nerve lesions with a subsequent weakening of the medius gluteus function is equally relevant.

A less invasive approach allowed to avoid the denervation of the gluteus muscles and, therefore, to expect a better strength in the post-operative period. This is also obtained because the gluteus maximus split is reduced compared to the typical K-L approach. A reduced muscular split gives a better hip abduction, as demonstrated by Dickson and Matta ${ }^{11,12}$.

After surgery, we noticed a decrease in blood loss due to a less invasive approach: mean blood loss of $200 \mathrm{ml}$ (range $100-400 \mathrm{ml}$ ). The classic K-L approach was associated with a mean blood loss of $900 \mathrm{ml}$ (range 100-3,500 ml) ${ }^{11}$. The ischiatic nerve was not challenged and it was not necessary to extend the incision to avoid damage to it. Even if the approach is minimally invasive, no dedicated devices were required. The radiographic and CT scan post-operative evaluation were good; in fact, the fractures healed, and there were no implants intraarticularly.

Addressing complications of this surgery, it is essential to avoid sciatic nerve injury. According to the literature, the iatrogenic sciatic lesion complication rate is $3-18 \%$ using the typical K-L approach. To prevent sciatic damage, we avoided using sharp devices closer to it and paid attention to patient positioning.

The appropriate approach to reduce the posterior column fracture is a posterior approach; the most known are the K-L or Gibson-Moore. These approaches were not compatible due to skin lesions. In the last twenty years, many approaches were described to decrease complications related to the K-L approach.

Carr and Leach, in 2006, described a small incision surgical exposure for selected fracture of the acetabulum defined as the gluteus maximus-splitting approach with lateral access ${ }^{5}$.

Perves described a posterior perigluteal approach with improved muscle tone and decreased rate of ossification ${ }^{10}$.

In 2014 A. Y. Sarlak et al. described the management of acetabular fractures with a modified posterior approach to spare external hip rotators; the incision started from the posterior inferior iliac spine and ends contouring the gluteus maximus. Therefore, it is not indicated in the treatment of acetabular fractures with skin abrasions ${ }^{3}$.
Moed BR, in 2014, published a modified Gibson approach; the approach is lateral, but it is possible to treat all the posterior acetabular fractures; it was possible to treat patients with posterior soft tissue damage ${ }^{8}$.

The patient we treated presented lesions that did not permit us to use the K-L approach. The technique described by Moed was not published yet, and the one described by Carr was not known to us; our choice was for an approach we were using since 2004 for the treatment of the posterior column fractures. This approach allowed us to reduce posterior wall and column fractures and avoid contamination due to close skin lesions.

Heterotopic ossifications (HO) occurred in 3\% of the patients with skin abrasions, and it is not correlated with the position of the lesions itself; Klein L. et al. ${ }^{13}$ and Dossett A.B. ${ }^{14}$ divide the burn injuries into two types: any associated fracture located outside the burned area or fractured bones within the burned area. This situation represents a critical factor that must be taken into account during treatment procedures. Patients with soft tissue damage in the fracture's site should be treated within 48 hours. We should consider the following options: there would be no difference in standard fracture treatment procedures in fractures outside the burned area. In our case, the skin lesion was not classifiable as inside or close to the fracture. Skin abrasion involved the dorsal part of the back and was extended beyond the posterior inferior iliac spine; the bruise was extended posteriorly on the thigh from the greater trochanter to the knee. We performed the surgery one week after the trauma.

Judet and Letournel describe an increased possibility to develop ossifications in patients affected by acetabular fractures with abdominal and head trauma in polytraumatized patients when associated with extended approaches. In our case report, the patient presented periarticular HO (Brooker type 3). The presence of the HO did not limit the hip range of motion. The ossifications were both in the anterior and posterior part without bridging the acetabulum and greater trochanter, which can cause hip ankylosis. Nowadays, Carr and Moed's approaches are the only ones used in skin lesions close to the acetabulum.

\section{Conclusion}

Treatment of posterior acetabular fractures is the most common in acetabular surgery but challenging to treat for inexperienced surgeons. Complications related to the K-L approach are well described in the literature; to improve patient outcomes, new procedures have been described to treat posterior acetabular fractures. In our case, a less invasive incision permitted us to treat this patient; knowing this approach or Moed's lateral approach can help to treat particular cases. 


\section{References}

1. Judet R, Judet J, Letournel E. Fractures of the acetabulum: classification and surgical approaches for open reduction. J Bone Joint Surg Am. 1964; 46: 1615-1646.

2. Letournel E. Acetabulum fractures: classification and management. Clin Orthop Relat Res. 1980; 151: 81-106.

3. Sarlak AY, Selek O, Inanir M, et al. Management of acetabular fractures with modified posterior approach to spare external hip rotators. Injury. 2014; 45(4): 732-737.

4. Magu NK, Rohilla R, Arora S, et al. Modified kocher-langenbeck approach for the stabilization of posterior wall fractures of the acetabulum. Journal of Orthopaedic Trauma. 2011; 25(4): 243249.

5. Carr JB, Leach PB. Small incision surgical exposure for select fracture of the acetabulum: the gluteus maximus-splitting approach. J Orthop Trauma. 2006; 20(8): 573-575.

6. Spagnolo R, Bonalumi M, Pace F, et al. Minimal-invasive posterior approach in the treatment of the posterior wall fractures the acetabulum. Chir Org Mov. 2009; 93(1): 9-13.
7. Moed BR. The modified Gibson posterior surgical approach to the acetabulum. J Orthop Trauma. 2010; 24(5): 315-322.

8. B.R. Moed. The modified Gibson approach to the acetabulum. Oper Orthop Traumatol. 2014; 26(6): 591-602.

9. Matta JM. Fractures of the acetabulum: accuracy of reduction and clinical results in patients managed operatively within 3 weeks after the injury. J Bone Joint Surg Am. 1996; 78(11): 1632-1645.

10. Perves A, Perreau M, Baufils P. Gluteal posterior approach for surgery of the acetabulum. Rev Chir Orthop Reparatrice Appar Mot. 1995; 81(7): 639-642.

11. Dickson WH, Duwelius PJ, Colville MR. Muscle strength testing following surgery for acetabular fractures. J Orthop Trauma. 1993; 7(1): 39-46.

12. Matta JM, Olson SA. Factors related to hip muscle weakness for lowing fixation of acetabular fractures. Orthopaedics. 2000; 23(3): 231-5.

13. Klein L, Dousa P, Zajicek R, et al. Specific aspect of the treatment of patients with multiple mechanical and burn injuries. Acta Chir Plast. 2008; 50(1): 17-22.

14. Dossett AB, Hunt JL, Purdue GF, et al. Early orthopedic intervention in burn patients with major fractures. J Trauma. 1991; 31(7): 888-92. 